\title{
Grupo Terapêutico com Idosos Sobre o Autocuidado nas Doenças Crônicas
}

\section{Therapeutic Group with Seniors about Self-Care in Chronic Diseases}

\author{
Murilo da Silva Alvesa; Maria da Conceição Filgueiras de Araújo ${ }^{\mathrm{a}}$; Mônica Pinchemel Nascimento ${ }^{\mathrm{b}}$; Francielli Cardoso \\ Ribeiro ${ }^{c}$; Patrícia Teixeira Rebouças ${ }^{c}$; Thalita Araújo dos Santos ${ }^{\mathrm{a}}$; Ariadne Nascimento Santos ${ }^{\mathrm{a}}$, Carilene Silva Oliveira ${ }^{\mathrm{a}}$; \\ Juliana Ingred de Oliveira ${ }^{\mathrm{d}}$ \\ aUniversidade Estadual de Santa Cruz; Departamento de Ciências da Saúde; Curso de Enfermagem. BA, Brasil.

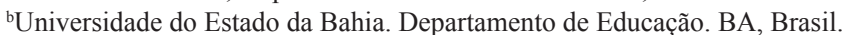 \\ 'Secretaria Municipal de Itabuna. BA, Brasil. \\ ${ }^{d}$ Universidade Estadual de Santa Cruz. Curso de Medicina. BA \\ *E-mail: \\ Recebido em: 11/11/2014; Aceito em: 04/05/2015
}

\begin{abstract}
Resumo
Diabetes e Hipertensão têm alta incidência mundial e associam-se à redução da longevidade e da qualidade de vida. A pesquisa objetivou descrever os conhecimentos de idosos em relação ao autocuidado, realizada em um grupo terapêutico de Diabetes e Hipertensão. Estudo qualitativo, descritivo, com nove idosos que participam do grupo terapêutico da Unidade de Saúde da Família de Itabuna-BA, realizados em seis momentos, sendo o primeiro um pré-teste com questionário semiestruturado (questões referentes ao perfil, noções sobre o autocuidado dos pacientes, conceitos e fatores desencadeantes da hipertensão e diabetes, importância do autocuidado, tratamento não farmacológico, complicações e prevenções das complicações das doenças crônicas abordadas), quatro encontros construídos com base nas lacunas do pré-teste e o pós-teste, com as mesmas questões. Os dados foram transcritos e analisados de acordo com a análise de Bardin. Surgiram duas categorias intituladas: reafirmando os conhecimentos prévios e a segunda, introduzindo os novos conhecimentos, sendo abordados os aspectos da etiologia, complicações, contribuição da atividade física, bem como a contribuição do grupo terapêutico em relação aos cuidados necessários ao tratamento. Como resultado, houve significativas mudanças no comportamento e na compreensão dos idosos em relação ao conhecimento destas doenças, estilo de vida adequado, hábitos alimentares saudáveis e as práticas de exercícios físicos. Grupos terapêuticos aparecem como possibilidade resolutiva frente ao controle, tratamento e autocuidado eficaz das doenças crônicas não transmissíveis.
\end{abstract}

Palavra-chave: Autocuidado. Terapêutica. Educação em Saúde. Doença Crônica.

\begin{abstract}
Diabetes and hypertension have high incidence worldwide and are associated with reduced longevity and quality of life. The research aimed to describe the elderly knowledge regarding to self-care, held in a Diabetes and Hypertension therapeutic group. Study qualitative, descriptive, involving nine elders participants of the therapeutic group ate the Unidade de Saude da Familia in Itabuna-BA, conducted into six times, the first being a pre-test with semi-structured questionnaire (issues relating to the profile, notions of self-care of patients, concepts and triggering factors of hypertension and diabetes, importance of self-care, non-pharmacological treatment, complications and their prevention of the complications of chronic diseases covered), four meetings built on the basis of gaps in the pretest and post-test, with the same issues. The data were transcribed and analyzed according to the analysis of Bardin. There were two categories titled: reaffirming the existing knowledge and the second by introducing the new knowledge being addressed to the aspects of etiology, complications, contribution of physical activity as well as the contribution of the therapeutic group, regarding to the care necessary for the treatment. As a result, significant changes in the behavior and understanding of the elderly, in relation to knowledge of these diseases, proper lifestyle, healthy eating habits and exercise practices. Therapeutic groups appear as a resolutive possibility facing the control, treatment and effective self-care of chronic non-communicable diseases.
\end{abstract}

Keywords: Self Care. Therapeutics. Health Education. Chronic Disease.

\section{Introdução}

A melhoria dos serviços de saúde, o aumento do nível de instrução e a concomitante evolução da medicina estão entre os principais fatores que contribuem para a transição epidemiológica no Brasil e no mundo. Aliado a estes fatores está o fenômeno mundial do envelhecimento da população, decorrente do declínio da mortalidade e da baixa taxa de fecundidade. Com a longevidade dos idosos, a saúde deste grupo etário é cada vez mais fonte de preocupação e objeto de estudo, visando seu bem-estar e aumento da expectativa de vida ${ }^{1}$.

Com o aumento dos anos vividos, as doenças crônicodegenerativas são cada vez mais prevalentes entre os idosos ${ }^{1}$.
Essas representam o problema de saúde de maior magnitude e são responsáveis por $72 \%$ das mortes, o que atrai atenção para esse grupo de doenças, a exemplo da Diabetes Mellitus - DM e da Hipertensão Arterial Sistêmica - HAS².

A DM e a HAS são altamente frequentes em idosos, tornando-se fatores determinantes na morbidade e mortalidade desta população, com considerável incidência em todo o mundo, não somente pela diminuição da longevidade, mas também por comprometer a qualidade de vida. Em razão desses fatores, atrela-se a necessidade de aceitação por parte do idoso das mudanças de hábitos que são fundamentais e, em geral, devem ser estabelecidas continuamente ${ }^{3,4}$.

Com esta demanda, o papel do profissional de saúde se 
amplia e aperfeiçoa diante da necessidade de uma nova visão, em que a conscientização e a iniciativa de propostas cada vez mais humanizadas são fundamentais. A responsabilidade para proporcionar condições que favoreçam o atendimento das pessoas com estas doenças é primordial, na perspectiva de propiciar mudanças no controle delas ${ }^{4}$.

A formação de grupos terapêuticos são ferramentas que vêm sendo utilizadas nas novas práticas de cuidado. Esses grupos têm como prioridade a informação, sendo homogêneos somente no que se refere às enfermidades, respeitando a diversidade sociocultural, focados na integralidade do atendimento, na ampliação do vínculo entre profissionais da saúde e pessoas, na educação em saúde, no desenvolvimento da autonomia e na promoção do autocuidado. São estruturados em dinâmicas que envolvem uma escuta cuidadosa sobre os relatos de experiências do grupo e posterior reflexão e incentivo do desenvolvimento de senso crítico com propostas voltadas ao tema ${ }^{4}$.

Deste modo, espera-se que as pessoas com doenças crônicas participantes do grupo terapêutico criem condições de viabilizar ações de autocuidado e, consequentemente, de adesão ao tratamento. A adesão ao tratamento inclui todos os fatores terapêuticos e educativos relacionados às pessoas que visam melhorar suas condições clínicas, envolvendo aspectos ligados ao entendimento e à aceitação de sua condição de saúde. É uma adaptação ativa identificando fatores de risco nos hábitos da vida cotidiana e assumindo atitudes que favoreçam a qualidade de vida e o desenvolvimento da consciência para o autocuidado ${ }^{5}$.

Estratégias como foco na educação em saúde, atreladas à valorização dos saberes das pessoas sobre as doenças crônicas e seu tratamento, são fundamentais nas práticas dos profissionais de saúde. Assim, o objetivo deste trabalho foi descrever os conhecimentos de idosos em relação ao autocuidado, realizado em um grupo terapêutico de Diabetes e Hipertensão.

\section{Material e Métodos}

Estudo qualitativo, descritivo, desenvolvido na Unidade de Saúde da Família Simão Fitterman, no município de Itabuna, BA. O grupo analisado, formado por nove idosos cadastrados no Programa Hiperdia - Sistema de Cadastramento e Acompanhamento de Hipertensos e Diabéticos, os quais já participavam das reuniões do grupo terapêutico de idosos.

A coleta de dados se deu a partir de seis encontros do grupo terapêutico específico para esta temática. $\mathrm{O}$ primeiro momento iniciou-se com a coleta de dados, por meio de um pré-teste constituído de um questionário semiestruturado, contendo 23 questões referentes ao perfil (idade, sexo, renda, presença e tempo com as doenças crônicas, hábitos de vida, sendo estes tabagismo, etilismo e sedentarismo), noções e importância do autocuidado, conceitos e fatores desencadeantes de hipertensão e diabetes, tratamento não farmacológico e prevenções das complicações das doenças crônicas abordadas.

Com base nas questões abordadas, quatro encontros foram construídos em formato de oficinas visando suprir as lacunas detectadas no pré-teste. No sexto momento foi realizada uma segunda coleta de dados, mediante um pós-teste composto das mesmas questões do pré-teste, visando detectar se novos conhecimentos e hábitos de vida dos idosos foram apreendidos e/ou incorporados em seu cotidiano. Os encontros foram realizados quinzenalmente, no período de três meses.

$\mathrm{Na}$ transcrição e análise dos questionários foi utilizado o método de análise do conteúdo proposto por Lawrence Bardin, que consiste na análise dos núcleos de sentido, conceituado como o conjunto de categorias empíricas que povoam o campo semântico definidor do conceito, das causas, das associações e da significância do fenômeno estudado.

A análise dos núcleos de sentido foi realizada com base em leitura cautelosa com posterior interpretação, associandoos aos contextos em que foram produzidos. Os participantes foram nomeados como informantes e enumerados em algarismos arábicos de 1 a 9 (exemplo: i1, i2).

Esta pesquisa foi aprovada pelo Comitê de Ética em Pesquisa da Universidade Estadual de Santa Cruz, sob o parecer número (protocolo n ${ }^{\circ}$ 307.145) CAAE: 3408713.5.0000.5526, que teve a integração dos discentes bolsistas do Programa de Educação pelo Trabalho para a Saúde (PET-Saúde) articulado ao Programa Nacional de Reorientação da Formação Profissional em Saúde - Pró-Saúde.

\section{Resultados e Discussão}

Os nove idosos participantes do grupo terapêutico implantado na unidade estão na faixa etária de 64 a 84 anos, sendo $66,6 \%$ do sexo feminino, $77,7 \%$ são aposentados, $88,8 \%$ recebendo entre um e dois salários mínimos, 77,7\% portadores de hipertensão arterial e $22,2 \%$ portadores de hipertensão e diabetes, sendo que $66,6 \%$ relataram tempo de doença maior que 10 anos.

Com relação aos hábitos de vida, $66,6 \%$ não são tabagistas, $55,5 \%$ relataram que não são etilistas e $66,6 \%$ dos indivíduos são sedentários.

Das questões que abordavam medidas de controle da doença e dieta recomendada, foram unânimes em responder ter recebido informações e incentivo dos Agentes Comunitários de Saúde - ACS, da enfermeira, do médico, dos familiares e amigos. Constatou-se que a participação destes grupos no processo de educação em saúde colabora com a valorização do conhecimento das pessoas sobre suas doenças crônicas e a adesão ao tratamento ${ }^{6}$.

Em alguns aspectos interrogados, os idosos relataram seus conhecimentos embasados nas suas experiências e conceitos prévios ao grupo terapêutico. Não obstante, pode-se identificar que novos conhecimentos foram adquiridos pelos idosos após a realização dos encontros do grupo terapêutico. 
Com todos esses resultados, surgiram duas categorias emergentes: reafirmando os conhecimentos prévios e introduzindo os novos conhecimentos.

\subsection{Reafirmando os conhecimentos prévios}

Esta categoria se refere ao conhecimento dos sinais e sintomas das doenças crônicas abordadas. Em relação a esse aspecto, 77,7\% $(\mathrm{n}=7)$ dos participantes declararam conhecêlos, sendo citados como principais sintomas dor de cabeça, tontura, alterações visuais e fraqueza. Tais conhecimentos foram identificados nas respostas dos informantes: "No começo fica tonta, dor na cabeça, na nuca e dá uma suadeira, aí sei que tá alta [...]”;(i1); “[...] Sim. Dor de cabeça, dor nas pernas, tontura, vistas escurecidas [...]” (i3).

O conhecimento da sintomatologia facilita o diagnóstico e a adesão ao tratamento, visto que significativa variedade das doenças crônicas evolui de forma insidiosa e subclínica ${ }^{4}$.

\subsection{Introduzindo os novos conhecimentos}

Para a construção desta categoria foram agregadas as seguintes subcategorias: etiologia, complicações, contribuição da atividade física para o tratamento e cuidados necessários para o controle das doenças crônicas. Esta escolha considerou que as subcategorias compreendiam a evocação do mesmo núcleo de sentido de novos conhecimentos, emergentes dentro do grupo.

Importante considerar que, após os encontros do grupo terapêutico, houve aumento de $33,3 \%$ de idosos que demonstraram ter apreendido acerca da etiologia da(s) sua(s) doença(s). Compreendeu-se que algumas etiologias apontadas pelos informantes como desencadeantes das doenças crônicas envolvem a hereditariedade, excesso no consumo de sódio e estresse. "Veio de família, minha mãe era, meu irmão e irmã também eram e depois as preocupações" (i1); "Acho que foi comida bem puxada no sal” (i2).

É de fundamental importância o conhecimento da etiologia $\mathrm{da}(\mathrm{s})$ doença(s) na adesão ao tratamento, pois permite o controle dos fatores de risco modificáveis (alimentação, sedentarismo, tabagismo, uso de álcool, entre outros), a promoção da saúde e a prevenção de complicações ${ }^{7}$.

No que se refere às complicações da hipertensão e da diabetes, nota-se que $44,4 \%(n=4)$ dos idosos não as conheciam. Ao término dos encontros, observou-se que os informantes da pesquisa descreviam as complicações com maior clareza, quando comparadas ao pré-teste, a exemplo: "Nervoso, raiva, não sei se é isso mesmo" (Pré-teste. i7); "Derrame, infarto [...]" (Pós-teste. i7).

Agregar conhecimentos influencia na adesão e no seguimento do tratamento, principalmente no que se refere à prevenção das suas complicações, sejam estas agudas ou crônicas, no autocuidado e na manutenção do controle metabólico e pressórico ${ }^{8}$.

Outro ponto importante das medidas de controle e auxílio ao tratamento é a atividade física: $77,7 \%(\mathrm{n}=7)$ dos informantes passaram a considerar a atividade física relevante: "Porque vai movimentar o corpo" (i4); "Porque o sangue desenvolve, circula" (i7); "Melhora o tratamento" (i5).

A prática regular de exercícios físicos é recomendada para todos os hipertensos e diabéticos, ela reduz a pressão arterial, os níveis glicêmicos e auxilia na redução ou manutenção do peso corporal. Além disso, o exercício físico pode reduzir o risco de doença arterial coronariana, acidente vascular cerebral e infarto agudo do miocárdio, como também auxilia na promoção da melhoria do bem-estar e do humor ${ }^{8}$.

Quanto aos cuidados necessários, a percepção dos informantes foi modificada em 77,7\% $(n=7)$, passando a considerar como fatores para o controle das doenças crônicas o uso regular da medicação, acompanhamento na unidade, abstenção do álcool e da nicotina, exercício físico regular e o controle do estresse e da alimentação: "Não comer sal, tomar os remédios" (i9); "Alimentação, medicação" (i5); "Tomar a medicação. Evitar o stress e relaxar" (i6); "Não tomar raiva, porque a raiva faz a pressão subir, conviver sempre bem com a vida" (I2); "Medir pressão, frequentar a unidade para trocar de medicação, alimentação" (I5).

Ao observar as falas dos usuários percebe-se a existência de contribuição exitosa por parte das oficinas. Com base na troca de saberes entre profissionais e usuários do serviço, os participantes do grupo terapêutico passaram a entender a importância da terapêutica medicamentosa e utilizá-la de forma adequada, visando garantir o sucesso no tratamento. "Orientação de como tomar os remédios. Vim aqui ajudou muito, graças a Deus!” (i1). "Tomar os remédios no horário certo $[\ldots]$ (i2).

A compreensão dos informantes sobre as condutas terapêuticas de controle da HAS e da DM estão de acordo com os estudos sobre essa temática, sendo análogas ao estilo de vida saudável: uso controlado do sal, da gordura, dos carboidratos e do açúcar, abstenção do álcool e da nicotina, exercício físico regular, controle do estresse e uso regular da medicação $0^{9,10}$.

O tratamento medicamentoso para HAS e DM objetiva originalmente a redução da morbimortalidade por condições cardiovasculares. Tem por finalidade a redução e/ou manutenção dos níveis pressóricos e glicêmicos em valores capazes de amenizar o risco para o desenvolvimento de complicações cardiovasculares, as quais contribuem para o aumento da mortalidade ${ }^{11,12}$.

Ao longo das oficinas, foram trabalhadas também temáticas relacionadas ao tratamento não farmacológico de ambas as doenças crônicas, que são importantes para controlar os níveis glicêmicos e pressóricos e/ou potencializar o efeito do tratamento medicamentoso de forma geral. Os achados demonstram que houve significativa apreensão dos conteúdos abordados. "Passei a controlar mais o sal. Sei das coisas que me faz mal" (i7); "Explicou coisas sobre a alimentação e 
ginástica e os cuidados para quem tem pressão alta" (i4).

Deve-se considerar que o tratamento não medicamentoso, como controle de peso e estresse, hábitos alimentares adequados, redução do consumo de sal e álcool, cessação do tabagismo, bem como estímulo à atividade física são importantes decisões para o controle de doenças crônicas. Contudo, deve-se considerar também a inclusão do suporte da equipe de saúde ${ }^{12-14}$.

\section{Conclusão}

Reconhecer a contribuição do grupo terapêutico para a melhoria do autocuidado em relação à HAS e ao DM é fundamental. A análise dos discursos dos usuários evidencia mudanças relevantes frente aos aspectos pesquisados.

A educação em saúde, ao respeitar as experiências e os saberes das pessoas envolvidas nas ações do grupo terapêutico, recupera articulações existentes, agrega valores, permite mudanças significativas na compreensão e no controle das doenças crônicas por parte dos idosos, como pontos primordiais para o desenvolvimento do autocuidado.

Conclui-se com esta pesquisa a real necessidade de haver espaços para que os idosos possam se expressar com base em seus conhecimentos a respeito das suas doenças, bem como ter acesso às informações necessárias, por meio dos profissionais de saúde, para o controle, prevenção e promoção da sua saúde e do bem-estar.

\section{Agradecimentos}

Ao Programa de Educação pelo Trabalho para a Saúde (PETSaúde) e ao Programa Nacional de Reorientação da Formação Profissional em Saúde (Pró-Saúde).

\section{Referências}

1. Rouquayrol MZ, Silva MGC. Rouquayrol epidemiologia \& saúde. Rio de Janeiro: MedBook; 2013.

2. Brasil. Ministério da Saúde. Plano de ações estratégicas para o enfrentamento das doenças crônicas não transmissíveis (DCNT) no Brasil. Brasília: MS; 2011.
3. Freitas EV, Py L. Tratado de geriatria e gerontologia. Rio de Janeiro: Guanabara Koogan; 2011.

4. Melo MC, Souza AL, Leandro EL, Mauricio EA, Silva ID, Oliveira JMO. A educação em saúde como agente promotor de qualidade de vida para o idoso. Ciênc Saúde Coletiva 2009;14(1):1579-86.

5. Fortuna CM, Matumoto S, Pereira MJB, Camargo-Borges C, Kawata LS, Mishima SM. Educação permanente na estratégia saúde da família: repensando os grupos educativos. Rev Latinoam 2013;21(4):990-7.

6. Girotto E, Andrade SM, Cabrera MAS, Matsuo T. Atenção ao tratamento farmacológico e não farmacológico e fatores associados na atenção primária da hipertensão arterial. Cienc Saúde Coletiva 2013;18(6):1763-72.

7. Acioli S, David HMSL, Faria MGA. Educação em saúde e a enfermagem em saúde coletiva: reflexões sobre a prática. Rev Enferm UERJ 2012;20(4):533-6.

8. Nogueira IC, Santos ZMSA, Mont'Alverne DGB, Martins $\mathrm{ABT}$, Magalhães CBA. Efeitos do exercício físico no controle da hipertensão arterial em idosos: uma revisão sistemática. Rev Bras Geriatr Gerontol 2012;15(3):587-601.

9. Figueiredo NN, Asakura L. Adesão ao tratamento antihipertensivo: dificuldades relatadas por indivíduos hipertensos Acta Paul Enferm 2010;23(6):782-7.

10. Cunha PRMS, Castelo Branco DR, Bernardes ACF, Aguiar MIF, Rolim ILTP, Linard AG. Prevalência e causas de não adesão ao tratamento anti-hipertensivo de idosos na atenção básica. Rev Pesq Saúde 2012;13(3):11-6.

11. Oliveira JEP, Vencio S. Diretrizes da Sociedade Brasileira de Diabetes: 2013-2014/Sociedade Brasileira de Diabetes. São Paulo: AC Farmacêutica; 2014.

12. Sociedade Brasileira de Cardiologia. Sociedade Brasileira de Hipertensão. Sociedade Brasileira de Nefrologia. VI Diretrizes Brasileiras de Hipertensão. São Paulo: ANAD; 2015.

13. Santos HLBA, Nascimento FMR, Freitas CMSM, Cunha AEV, Vancea DMM. Motivação do diabético tipo 2 para o tratamento não farmacológico. Rev Bras Ativ Fis Saúde 2012;17(6):485-94.

14. Fortuna CM, Matumo S, Pereira MJB, Mishima SM, Kawata LS, Camargo-Borges C. O enfermeiro e as práticas de cuidados coletivos na estratégia saúde da família. Rev Latinoam Enferm 2011;19(1):581-8. 